\title{
RESEARCH HIGHLIGHT Ultrasensitive antigen density discrimination by synNotch
}

\author{
Divanshu Shukla ${ }^{1}$ and James L. Riley (iD) \\ Cell Research (2021) 31:725-726; https://doi.org/10.1038/s41422-021-00511-y
}

\begin{abstract}
In a recent study published in Science, Hernandez-Lopez et al. engineered a low-to-high two-step regulatory circuit that allows $T$ cells to discriminate target antigens with an ultrasensitive threshold. In this circuit, low-affinity synNotch receptor controls the expression of the high-affinity chimeric antigen receptor (CAR); thus, the only time the CAR construct is expressed is in the presence of high levels of antigen, preventing off-tumor CAR T cell recognition.
\end{abstract}

Chimeric antigen receptor (CAR) $T$ cells have shown an impressive ability to cure CD19-expressing tumors, but these engineered T cells invariably have "on-target, off-tumor" toxicity that injure the normal tissues, hindering the development of CAR $T$ cells targeting other tumors. Indeed, there are significant ontarget, off-tumor toxicities associated with CD19-specific CARs, with the most noticeable victim being $B$ cells. Fortunately, there are therapeutic options to minimize the loss of these immune cells. However, CD19 expression has recently been detected in brain mural cells, which may be related to the neurotoxicity observed in some individuals. ${ }^{1}$ This suggests that there are no unique tumor markers or antigens expressed only on "dispensable" tissue, but rather a collection of proteins with extracellular domains that are expressed to varying degrees primarily on tumors and less so on healthy tissues. ${ }^{2}$

Several strategies have been put forward to focus CAR T cells on the tumor while paying less attention to healthy tissue. In one report, pre-treatment with CAIX mAb blocks the access of engineered T cells to CAIX expressed in bile duct epithelial cells, reducing on-target, off-tumor toxicity of CAIX CAR T cells. ${ }^{3}$ In another strategy, an antagonistic receptor system, inhibitory CARs (iCARs), were engineered by replacing the cytoplasmic signaling domains with inhibitory signaling chains of the negative regulators of $\mathrm{T}$ cell activation, $\mathrm{PD}-1$ or $\mathrm{CTLA}-4$. In a preclinical study, iCAR allows $T$ cells to discriminate the target antigen from the bystander cells and limit off-tumor cytokine secretion and cytotoxicity. ${ }^{4}$ While these approaches have promise, they try to limit the activity of a fully competent CAR T cell, which may prove challenging to do on a long-term basis. The synNotch receptor, on the other hand, was developed to only allow expression of a fully functional CAR construct under the right environmental cues. Here, the extracellular Notch domain was replaced by sensor module (e.g., ScFv) and the intracellular domain tethers a transcriptional regulator. Upon engagement of target antigen with the synNotch receptor, an orthogonal transcription factor (e.g., TetR-VP64 or Gal4-VP64) is cleaved from cytoplasmic tail in an ADAM and $\gamma$-secretase dependent manner and this transcription factor drives the expression of the functional CAR construct. ${ }^{5}$ In the original application of the synNotch receptor, $T$ cells were engineered to express the CAR only when another molecule was present. In this way, a tumor could be defined as tissue that simultaneously expressed both markers, and healthy tissue that expressed only one of these markers would be ignored by the CAR T cells.

What if there is not a combination of antigens that clearly distinguishes tumor from healthy tissue? How can the synNotch be used to control CAR expression to prevent off-tumor toxicity? In a recent study, Hernandez-Lopez et al. cleverly addressed this question. The authors hypothesized that a low-affinity antiHER2 synNotch receptor (scFv $K_{d}=210 \mathrm{nM}$ ) would only be triggered when there is high expression of HER2 on the surface of a cell, as in the case with tumors, and not triggered when there is low expression, as in the case with healthy tissues. ${ }^{6}$ Upon triggering, this low-affinity synNotch receptor would release a CAR-specific transcription factor that would induce expression of a high-affinity (scFv $K_{d}=17.6 \mathrm{nM}$ ) HER2 CAR. Their initial in vitro studies using tumor cell lines with increasing amounts of HER2 expression indicated that this receptor system functioned as an ultrasensitive response. A classic example of ultrasensitive response is observed for the IL-2 cytokine. Herein, binding of IL-2 to low-affinity receptor results in induced upregulation of high-affinity IL-2 receptors (IL-2R), which further activates the T cells and establishes a positive feedback. ${ }^{7}$ In the synNotch system described above, a flat line response to increasing amounts of low antigen was observed until a density of antigen was reached that triggered the synNotch receptor. At this point, there was a rapid increase in cytolytic activity, resulting in a sigmoidal curve that is archetypical for an ultrasensitive response (Fig. 1). To examine whether ultrasensitive detection could occur in a multi-cellular system, HernandezLopez et al. cultured low or high HER2-expressing human breast epithelial MCF10A cell lines with two-step circuit HER2-specific T cells described above and implanted them in Matrigel to make a three-dimensional (3D) target spheroid culture model. They observed that the synNotch T cells efficiently attacked, killed, and disassembled the high HER2-expressing spheroids but were unresponsive to low HER2-expressing spheroids. In contrast, unregulated CAR T cells had significant activity against both low and high HER2 density spheroids. Using a similar experimental design, Hernandez-Lopez and colleagues showed that this T cell design could function in vivo within humanized mice to destroy high HER2-expressing tumors and ignore low HER2-expressing tumors.

In conclusion, the ultrasensitive antigen-density discriminatory ability of synNotch-CAR engineered $T$ cells described by Hernandez-Lopez and colleagues is a promising avenue by which high CAR $T$ cell activity against tumors is maintained while minimizing on-target, off-tumor toxicity, providing that the heathy tissue expresses sufficiently low levels of the target antigen. Clinical trials ultimately will determine whether this approach

${ }^{1}$ Department of Microbiology, Center for Cellular Immunotherapies, University of Pennsylvania, Philadelphia, PA 19104, USA

Correspondence: James L. Riley (rileyj@upenn.edu)

Published online: 4 May 2021 
a

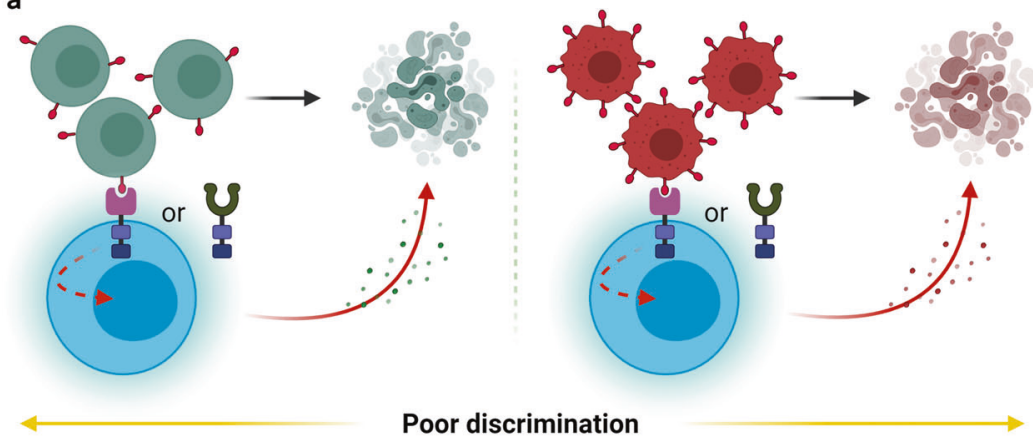

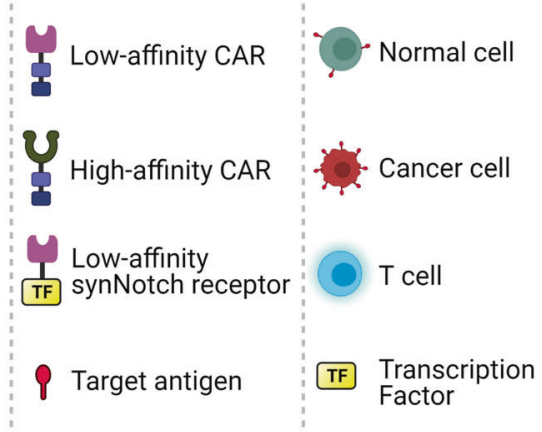

b Two-step synNotch-to-CAR circuit

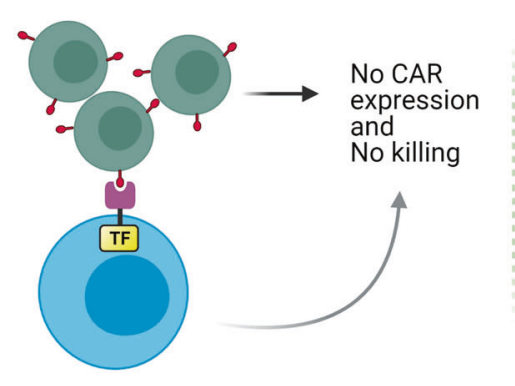

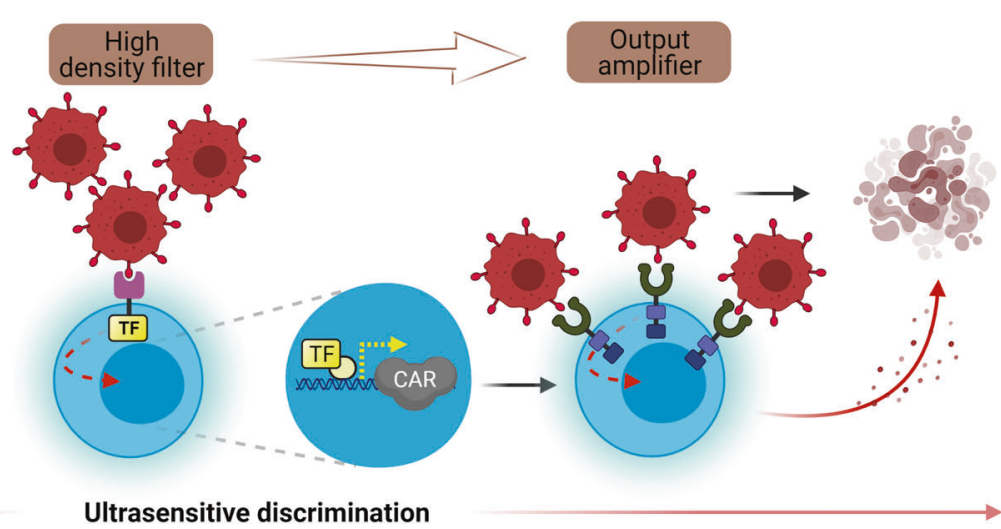

Fig. 1 Overview of synNotch CAR that becomes active only in the presence of high levels of antigen. a Based on the results shown by Hernandez-Lopez et al., ${ }^{6}$ constitutive CAR T cells expressing either low- or high-affinity CARs rapidly killed low (left) as well as high (right) antigen target cells, showing poor density discrimination. b Upon the interaction of low antigen density target, low-affinity synNotch receptors suppress the expression of CAR (left). Conversely, in the presence of high antigen density target, synNotch receptor enhances the expression of CAR and the T-cell activity is dominated by the high-affinity CAR response to eliminate the tumor cells and minimize on-target, off-tumor toxicities (right), showing an ultrasensitive antigen density response. Illustration was made in ${ }^{\circ}$ BioRender - biorender.com.

widens the therapeutic window of engineered $T$ cells against solid cancers and results in more FDA-approved therapies for CAR T cells.

\section{ADDITIONAL INFORMATION}

Competing interests: J.L.R. is a co-founder and shareholder of Tmunity Therapeutics. He has been in receipt of research funding from Tmunity Therapeutics. D.S. declares no competing interests.

\section{REFERENCES}

1. Parker, K. R. et al. Cell 183, 126-142 (2020).

2. Ellis, G. I., Sheppard, N. C. \& Riley, J. L. Nat. Rev. Genet. https://doi.org/10.1038/ S41576-021-00329-9 (2021).

3. Lamers, C. H. et al. Mol. Ther. 21, 904-912 (2013).

4. Fedorov, V. D., Themeli, M. \& Sadelain, M. Sci. Transl. Med. 5, 215 ra172 (2013).

5. Morsut, L. et al. Cell 164, 780-791 (2016).

6. Hernandez-Lopez, R. A. et al. Science 371, 1166-1171 (2021).

7. Busse, D. et al. Proc. Natl. Acad. Sci. USA 107, 3058-3063 (2010). 